\title{
Developing a Structural Health Monitoring System for Nuclear Dry Cask Storage Canister Xiaoyi Sun ${ }^{1}$, Bin Lin ${ }^{1}$, Jingjing Bao ${ }^{1}$, Victor Giurgiutiu ${ }^{1}$, Travis Knight ${ }^{1}$, Poh-Sang Lam ${ }^{2}$, Lingyu \\ ${ }^{1}$ Mechanical Engineering Department, University of South Carolina, Columbia, $\mathrm{SC}^{2}$ Savannah River National Laboratory, Aiken, South Carolina, USA
}

\begin{abstract}
Interim storage of spent nuclear fuel from reactor sites has gained additional importance and urgency for resolving waste-management-related technical issues. In total, there are over 1482 dry cask storage system (DCSS) in use at US plants, storing 57,807 fuel assemblies. Nondestructive material condition monitoring is in urgent need and must be integrated into the fuel cycle to quantify the "state of health", and more importantly, to guarantee the safe operation of radioactive waste storage systems (RWSS) during their extended usage period. A state-of-the-art nuclear structural health monitoring (N-SHM) system based on in-situ sensing technologies that monitor material degradation and aging for nuclear spent fuel DCSS and similar structures is being developed. The N-SHM technology uses permanently installed low-profile piezoelectric wafer sensors to perform long-term health monitoring by strategically using a combined impedance (EMIS), acoustic emission (AE), and guided ultrasonic wave (GUW) approach, called "multimode sensing", which is conducted by the same network of installed sensors activated in a variety of ways. The system will detect $\mathrm{AE}$ events resulting from crack (case for study in this project) and evaluate the damage evolution; when significant AE is detected, the sensor network will switch to the GUW mode to perform damage localization, and quantification as well as probe "hot spots" that are prone to damage for material degradation evaluation using EMIS approach. The N-SHM is expected to eventually provide a systematic methodology for assessing and monitoring nuclear waste storage systems without incurring human radiation exposure.
\end{abstract}

Keywords: Nuclear energy, dry cask canister, structural health monitoring, piezoelectric, sensing

\section{INTRODUCTION}

In total, there are over 1482 dry cask storage system (DCSS) in use at US nuclear power plants (NPP), storing 57,807 fuel assemblies. Following the issuance of the Blue Ribbon Commission (BRC) final report on America's Nuclear Future in 2012, interim storage of spent nuclear fuel from reactor sites has gained additional importance and urgency for resolving waste-management-related technical issues. To ensure that nuclear power remains clean energy, safe, long-term management of used nuclear fuel and high level radioactive waste "remains a national priority".

Structural health monitoring (SHM) is an emerging technology that aims at monitoring the state of a structure through the use of networks of permanently mounted sensors ${ }^{[1]-[2]}$. SHM is an emerging technology that uses in-situ sensory system to perform rapid nondestructive detection of structural damage as well as long-term integrity monitoring. The permanently installed (embedded) sensors can probe the structures at any time over the entire service life, which is superior to the handheld conventional ultrasonic nondestructive testing (NDT) techniques. SHM technologies have been developed primarily within the aerospace and civil engineering communities. However, SHM concepts and methodologies could be extended to other safety-critical systems. The nuclear dry cask storage systems (DCSS) are safety-critical facilities in need of monitoring over prolonged periods of time. One of the key aspects of improving DCSS reliability, sustaining the safety, and extending the life of current PVP is to develop technologies that can better diagnose their state of structural health. SHM technology fits well with these requirements and has great potential for broad applications in the nuclear field. Combined with appropriate data analysis algorithms, SHM is expected to provide timely information regarding the structural integrity for condition assessment and diagnosis of components important to safety (ITS) in the DCSS at any time. The integration of a nuclear SHM system into a nuclear spent fuel storage system will facilitate the evaluation of degradation and aging of used nuclear fuel containers and storage facilities over extended storage periods. Eventually, it will ensure a systematic methodology for assessing and monitoring nuclear waste storage systems without incurring human radiation exposure.

Smart Materials and Nondestructive Evaluation for Energy Systems 2015, edited by Norbert G. Meyendorf, Proc. of SPIE Vol. 9439, 94390N · (C) 2015 SPIE · CCC code: 0277-786X/15/\$18 · doi: 10.1117/12.2085045 


\section{GUIDED WAVE MODEL WITH NUCLEAR EFFECTS}

This paper addresses the development of an in-situ SHM system for DCSS long term monitoring and evaluation based on the use of piezoelectric wafer active sensors (PWAS), with particular focus on some of the challenges associated with the nuclear effects on DCSS system. The primary goal of in-situ sensing of structural components is to reliably interrogate large areas and detect structural anomalies. However, when applied to nuclear environment, even the relatively less harsh DCSS situation, prolonged exposure to nuclear radiation as well as elevated temperature may introduce measurement artifacts and significant damage to both sensors and the sensing system. In fact, the effects of nuclear environments on this technology are not yet well addressed and understood. It is of paramount importance to assess and understand how the piezoelectric ultrasonic SHM systems are affected by nuclear radiation and high temperature factors; thus to protect them when necessary. We studied the nuclear temperature effects on PWAS preliminarily through the evaluation of the electromechanical impedance response. Piezoelectric Wafer Active Sensors

The key technology to an effective N-SHM system is the sensing element that can detect the degradation under the harsh nuclear DCSS environment. The past two decades have witnessed an extensive development of SHM sensor technology ${ }^{[3]-[5]}$. A wide range of sensors have been developed particularly for generating and receiving acoustoultrasonic waves. PWAS have emerged as one of the major SHM technologies ${ }^{[6]-[7]}$. A variety of damage detection methods can be applied to this type of sensor: (a) propagating ultrasonic waves, both acoustic emission (AE) ${ }^{[8]}$ and guided ultrasonic waves $(\mathrm{GUW})^{[9]}$; and (b) standing ultrasonic waves, i.e., electromechanical impedance spectroscopy ${ }^{[10]}$ (EMIS) as illustrate in Figure 1and Figure 2.

- $\quad$ AE for crack initiation has been shown to enable the detection of crack initiation and crack progression; $\mathrm{AE}$ provides earlier warning of impending damage than any other methods. Because it is very sensitive to damage events, the AE method has been used for many applications in aerospace and civil engineering applications.

- GUW quantitative damage detection and evaluation relies on in interrogative ultrasonic waves propagating and reflecting within the structure to identify wave field disturbances due structural damage and flaws. An N-SHM system using interrogative GUW would be able to cover large areas from one single location, thereby being cost-effective and time-efficient. Research on embedded PWAS GUW-SHM has been conducted nationally and internationally for damage detection on both metallic and composite thin-walled structures.

- $\quad$ EMIS for local material degradation monitoring: is considered a promising approach for PWAS structural NDE. This method utilizes high frequency structural excitations, which are typically higher than $30 \mathrm{kHz}$ through surface-bonded PWAS to monitor changes in the structural E/M impedance. Previous studies have confirmed that EMIS is sensitive to very small amounts of material changes, suggesting that EMIS offers the potential for detection of the progression of small damage at the material-level in a metallic material.

Our previous research has successfully demonstrated the use of PWAS on thick steel components and investigated the durability and survivability of the PWAS transducers under various exposures (cryogenic and high temperature, temperature cycling, freeze-thaw, outdoor environment, operational fluids, large strains, fatigue load cycling ${ }^{[11]}$. In most cases, the PWAS survived the tests successfully. The cases when the PWAS did not survive the tests were closely examined and possible cause of failure was discussed. The test results indicated that PWAS can be successfully used in cryogenic environment; however, it does not seem to be a good candidate for high temperature. Repeated differential thermal expansion and extended environmental attacks can lead to PWAS failure. This emphasizes the importance of achieving the proper design of the adhesive bond between the PWAS and the structure, and of using protective coating to minimize the ingression of adverse agents. Our preliminary work on the effects of nuclear radiation on PWAS exposed to irradiation effects published in $2012^{[12]}$. We have discovered that the short term irradiation affected the capacitance and the electromechanical impedance of PWAS. A predictive modeling on PWAS under radiation effect for DCSS system is necessary. 


\section{Propagating Lamb waves}

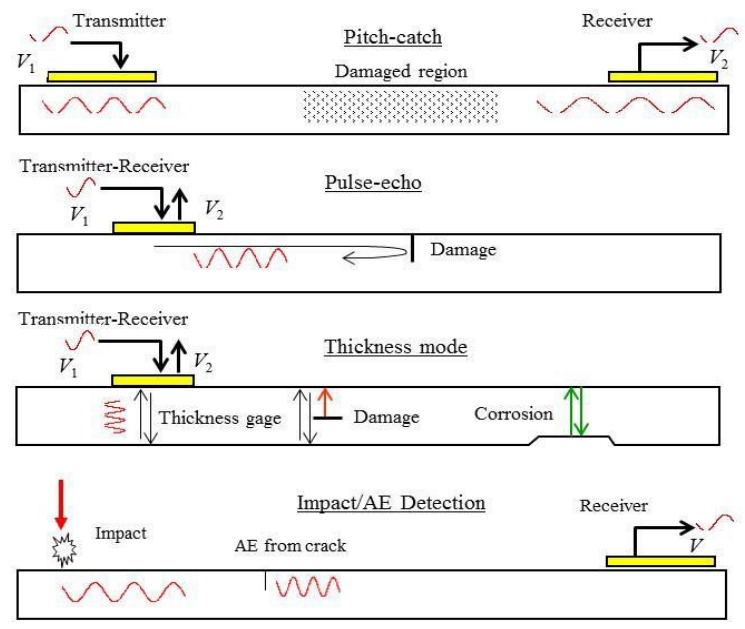

Figure 1. The PWAS are used for structural sensing with propagating ultrasonic guided waves. The propagating wave methods include: pitch-catch; pulse-echo; thickness mode; and passive detection of impacts and acoustic emission (AE).
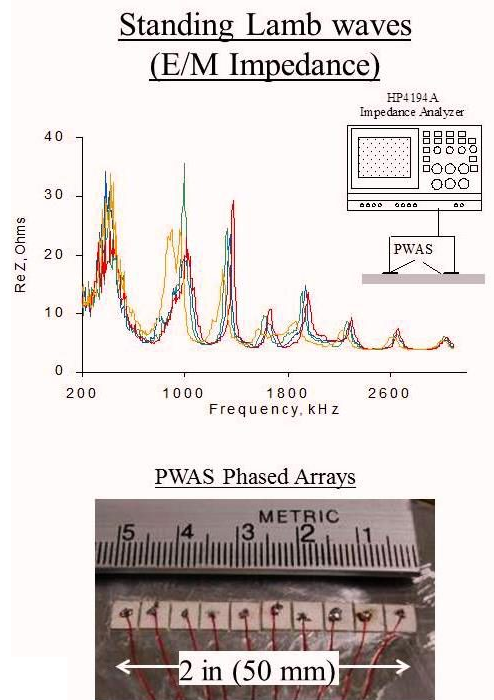

Figure 2. The PWAS are used for structural sensing includes standing waves and phased arrays.

\section{ELECTROMECHANICAL IMPEDANCE BASICS}

For a linear piezoelectric material, the relation between the electrical and the mechanical variables can be described by linear relations:

$$
\left[\begin{array}{l}
S \\
D
\end{array}\right]=\left[\begin{array}{ll}
s^{E} & d_{t} \\
d & \varepsilon^{T}
\end{array}\right]\left[\begin{array}{l}
T \\
E
\end{array}\right]
$$

where $S$ is the mechanical strain; $T$ is the mechanical stress; $E$ is the electric field; $D$ is the charge density; $s$ is the mechanical compliance; $d$ is the piezoelectric strain constant; and $\varepsilon$ is the permittivity. The superscripts $E$ and $T$ indicate that those quantities are measured with electrodes connected together and zero stress, respectively, and the subscript $t$ 
indicates transpose. The first equation describes the converse piezoelectric effect and the second describes the direct effect:

$$
Z_{s t r}(\omega)=k_{s t r}(\omega) / j \omega=k_{e}(\omega)-\omega_{m}^{2}(\omega)+j \omega c_{e}(\omega)
$$

Through the mechanical coupling between PWAS and the host structure, on one hand, and through the E/M transduction inside the PWAS, on the other hand, the drive-point structural impedance is reflected directly in the electrical impedance, $Z(\omega)$, at the PWAS terminals:

$$
Z(\omega)=\left[j \omega C\left(1-\kappa_{31}^{2} \frac{\chi(\omega)}{1+\chi(\omega)}\right)\right]^{-1}
$$

where $\mathrm{C}$ is the zero-load capacitance of the PWAS and $\kappa_{31}$ is the E/M cross coupling coefficient of the PWAS $\left(\kappa_{31}=d_{31} / \sqrt{\bar{s}_{11} \bar{\varepsilon}_{33}}\right)$, and $\chi(\omega)=k_{s t r} / k_{P W A S}$ with $k_{P W A S}$ being the static stiffness of the PWAS.

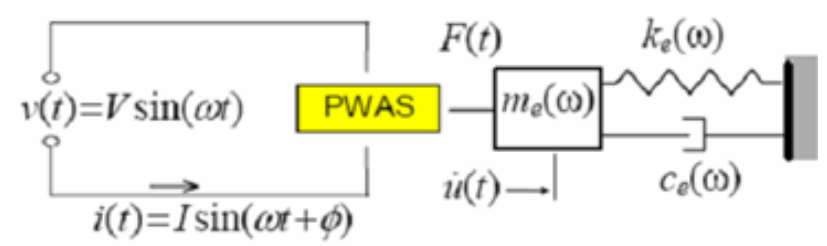

Figure 3. E/M coupling between PWAS and structure for a 1-D dynamic model

\section{TEMPERATURE EFFECTS ON E/M IMPEDANCE}

We first investigated the temperature effects on free PWAS through the evaluation of the electromechanical (E/M) impedance response at elevated temperatures.

\subsection{Temperature testing setup}

The temperature tests are performed in a well-controlled oven (Quincy Lab Inc. lab oven, model $40 \mathrm{GC}$, highest temperature $232^{\circ} \mathrm{C}$ with $3{ }^{\circ} \mathrm{C}$ stability) in the laboratory. A fixture has been designed and developed for holding two test PWAS inside the oven and for in-situ impedance measurement. The fixture is shown in Figure 4(a). The PWAS was held by two pogo pins (zoom-in provided in Figure 4a) with one end of the pin in touch with the PWAS electrode and the other having wiring extension. The fixture, with the PWAS being tested, were then put in the oven with wires coming out of a hole on top of the oven, as indicated in Figure $4 \mathrm{~b}$. The temperatures were set and read by a temperature controller and a thermocouple (Figure 4c), respectively. The temperature controller consists of a thermocouple (K type) and a microcontroller (Yudian 518) to measure and set up the temperature of the oven, with the thermocouple being placed in the oven through another hole on the top of the oven, as shown in Figure 4c. Testing temperature started with the room temperature at $22{ }^{\circ} \mathrm{C}$ and stopped at $225^{\circ} \mathrm{C}$, with an increment of $25^{\circ} \mathrm{C}$. Data were taken when temperature controller gave a stable value. Impedances of free PWAS at the frequency range from $200 \mathrm{kHz}$ to $1 \mathrm{MHz}$ were measured with HP4194A impedance analyzer shown in Figure 4d. In the test, two PWAS (labeled as PWAS 1 and PWAS 2) were tested from room temperature to $225^{\circ} \mathrm{C}$ for a day (Day1), waiting for it to cool down and repeated around the same time on the second day (Day2). 


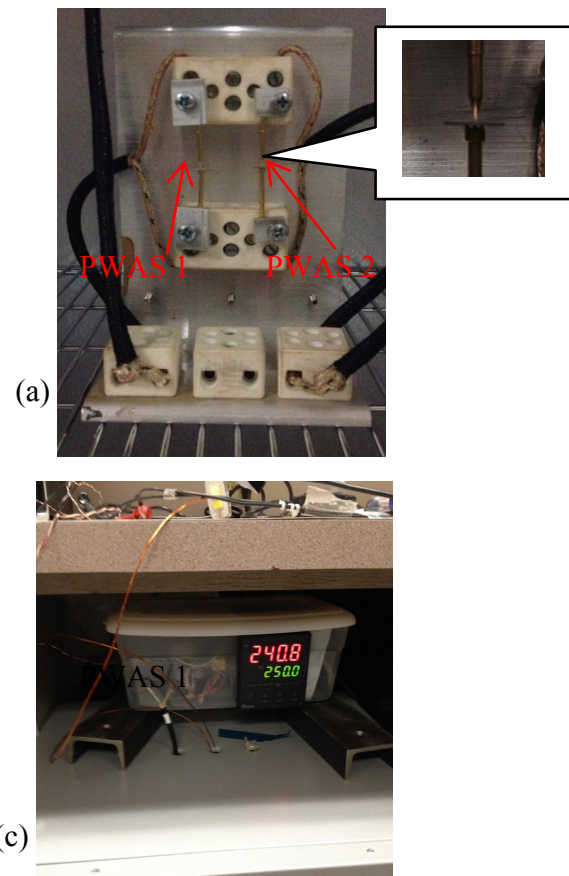

(b)

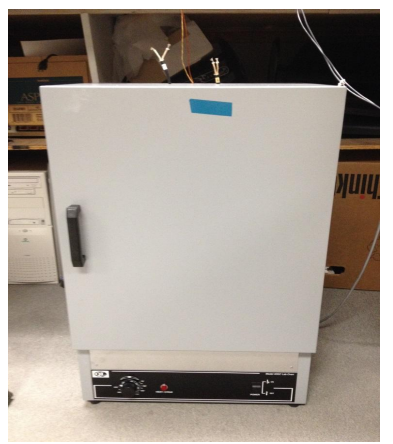

(d)

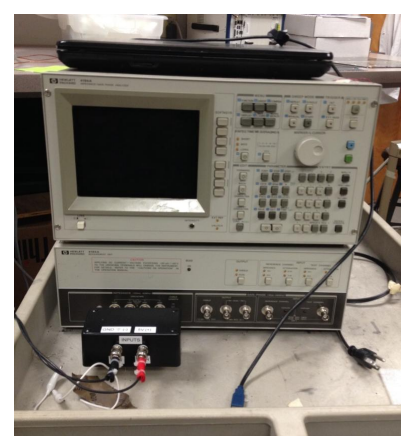

Figure 4. Experimental setup: (a) fixture with the PWAS in the oven; (b) lab oven as temperature chamber; (c) temperature controller setting and showing the temperature; (d) HP4194A for admittance and impedance measurement

\subsection{Testing results}

Impedances of the tested free PWAS within the frequency range from $200 \mathrm{kHz}$ to $1 \mathrm{MHz}$ have been recorded. The real parts of the PWAS impedance (ReZ) at different temperatures were plotted in Figure 5 and Figure 6 for Day 1 and Day 2, respectively. Within the tested frequency range, two resonant frequencies are observed, around $350 \mathrm{kHz}$ for the first resonance $\left(1^{\text {st }} \mathrm{Rs}\right)$ and $850 \mathrm{kHz}$ for the second resonance $\left(2^{\text {nd }} \mathrm{Rs}\right)$. The first two resonances have been studied in details and reported below.

To obtain more details for the resonances, zoom-in of the first resonances are provided in Figure 7 and Figure 8, respectively. Figure 7 and Figure 8 indicate that on Day 1, as the temperature rise, the first resonances gradually shift to the lower frequency end while on the Day 2 it gradually shift to the higher frequency as the temperature rise. Zoom-in of the second resonances are also acquired and provided in Figure 9 and Figure 10. The details show that on Day 1 as the temperature increase, the frequencies of the second resonances fluctuate at first but it eventually rise with the temperature, while on Day 2 the resonances monotonically shift to the higher frequency.

The amplitudes $\left(A_{\mathrm{ZR} 1}\right)$ and resonant frequencies $\left(f_{\mathrm{ZR} 1}\right)$ of the first impedance resonances at each temperature were further obtained and studied, respectively. Figure 11 presented that the amplitude of the first impedance resonance. The tendencies of how the parameters of both PWAS changed with the temperature stay almost the same. For the data acquired on the first day, the amplitudes fluctuate at the beginning but eventually decrease, as for the data obtained on the second day, at the beginning, the amplitude increase with the temperature rising, but after the temperature arrives at $150{ }^{\circ} \mathrm{C}$, decrease appears. Figure 12 provides the resonant frequencies of first impedance resonance. The change trend of both PWAS also accords with each other. The frequencies decrease monotonically and nearly linearly with the increase of the temperature on the first day while the frequencies increase on the second day. The frequencies and amplitudes of first resonance against temperature are listed in the Table 1and Table 2. 


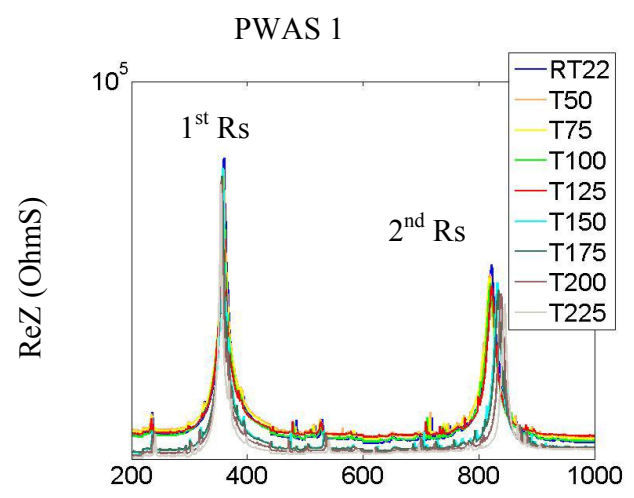

Frequency $(\mathrm{kHz})$

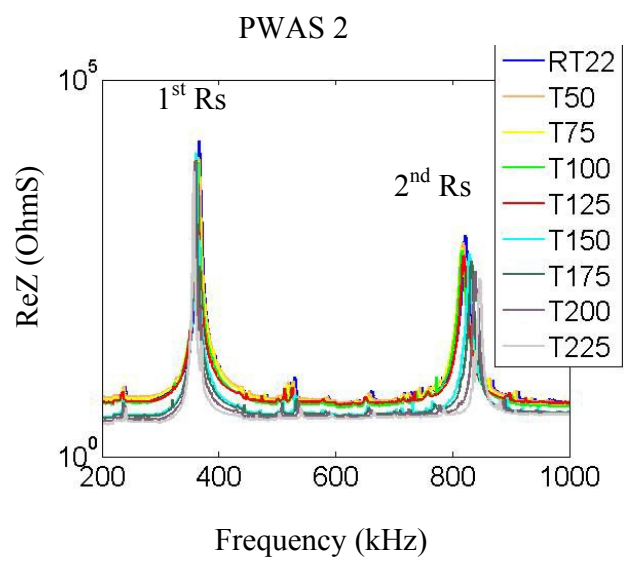

Figure 5. Real part impedance of PWAS (Day1): (a) PWAS 1; (b) PWAS 2.

PWAS 1

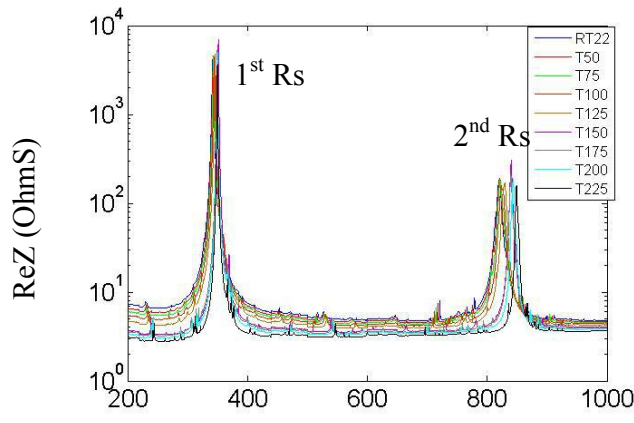

(a)
Frequency $(\mathrm{kHz})$
PWAS 2

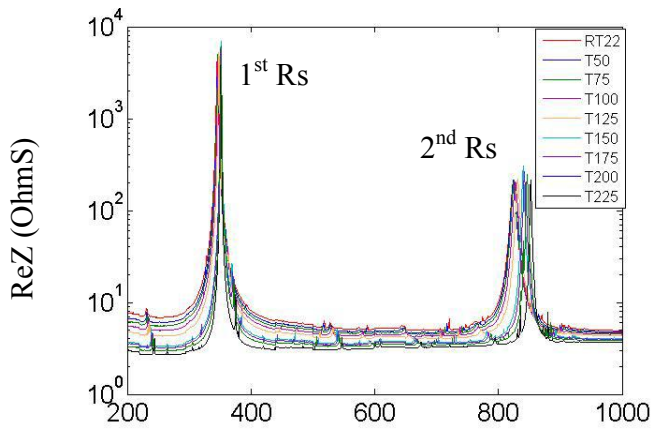

(b) Frequency $(\mathrm{kHz})$

Figure 6. Real part impedance of PWAS (Day2): (a) PWAS 1; (b) PWAS 2.

PWAS 1

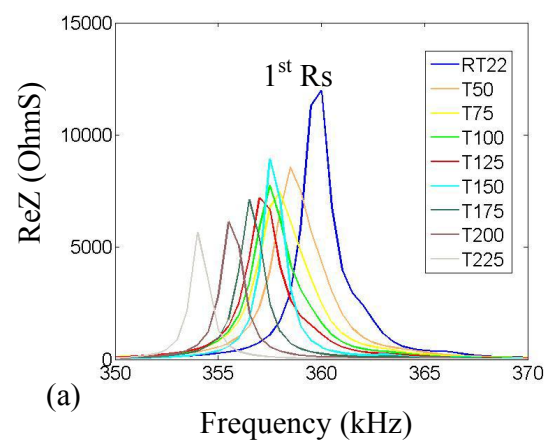

PWAS 2

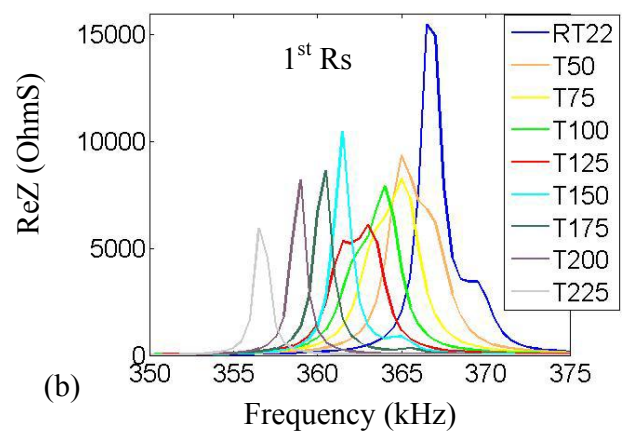

Figure 7. Zoom-in of first resonance from 350 to $370 \mathrm{kHz}$ (Day1): (a) PWAS 1; (b) PWAS 2 
PWAS 1

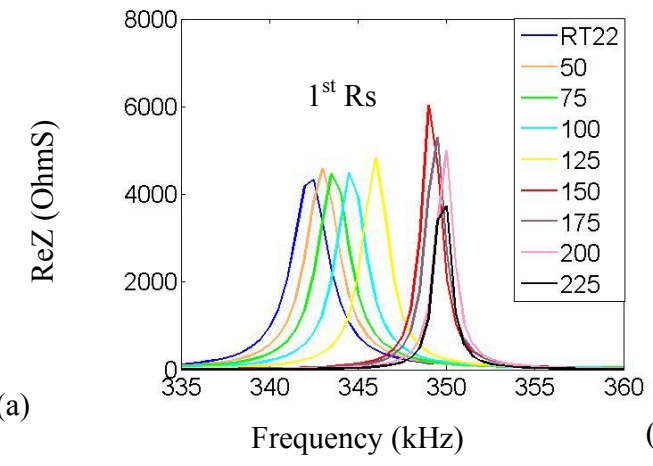

PWAS 2

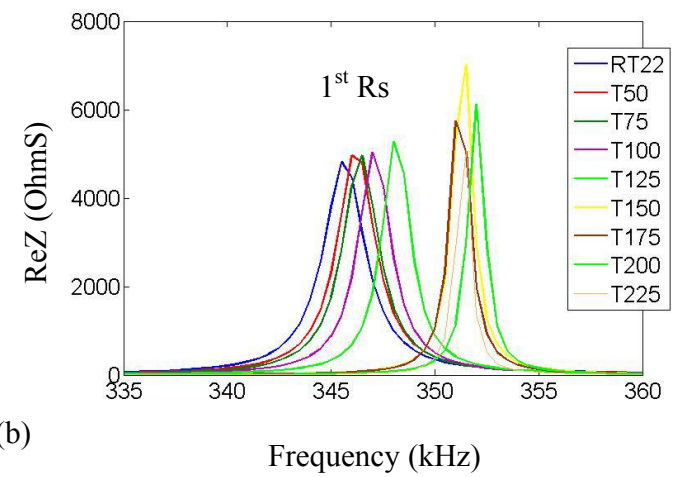

Figure 8. Zoom-in of first resonance from 335 to $360 \mathrm{kHz}$ (Day2): (a) PWAS 1; (b) PWAS 2

PWAS 1

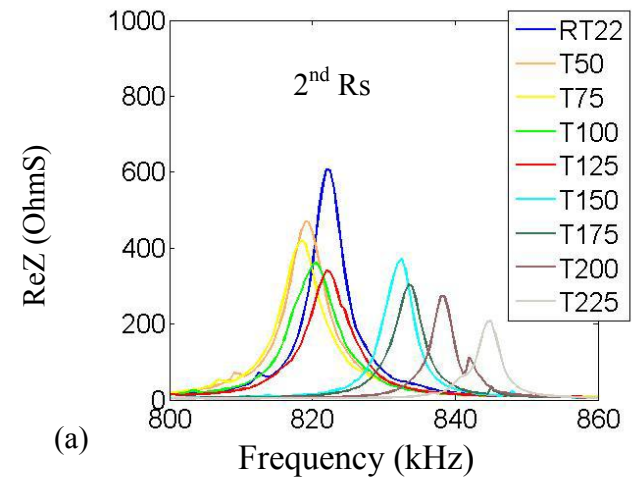

PWAS 2

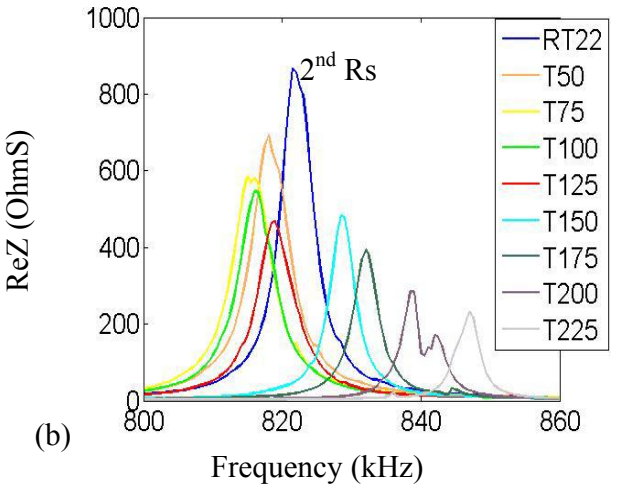

Figure 9. Zoom-in of second resonance from $800 \mathrm{kHz}$ to $860 \mathrm{kHz}$ (Day1): (a) PWAS 1; (b) PWAS 2
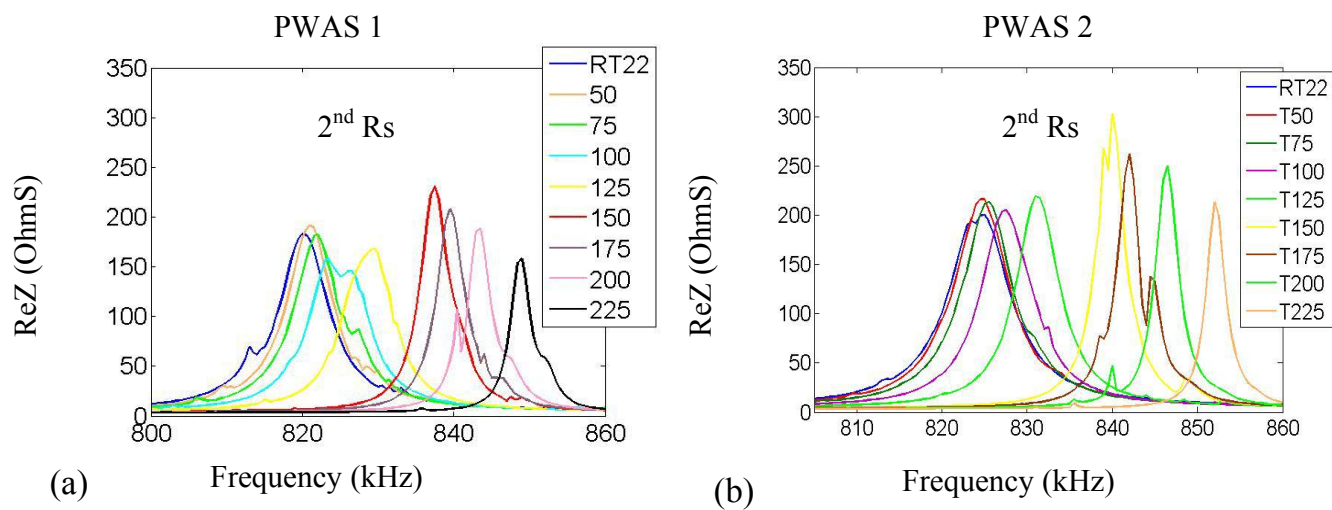

Figure 10. Zoom-in of second resonance from $800 \mathrm{kHz}$ to $860 \mathrm{kHz}$ (Day2): (a) PWAS 1; (b) PWAS 2. 
PWAS 1

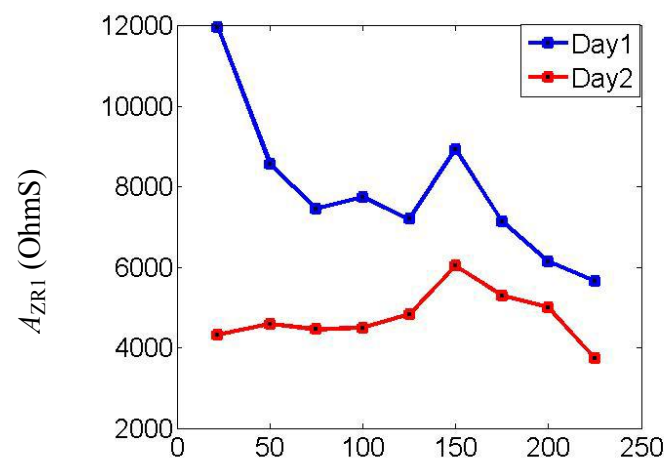

(a)

Temperature $\left({ }^{\circ} \mathrm{C}\right)$
PWAS 2

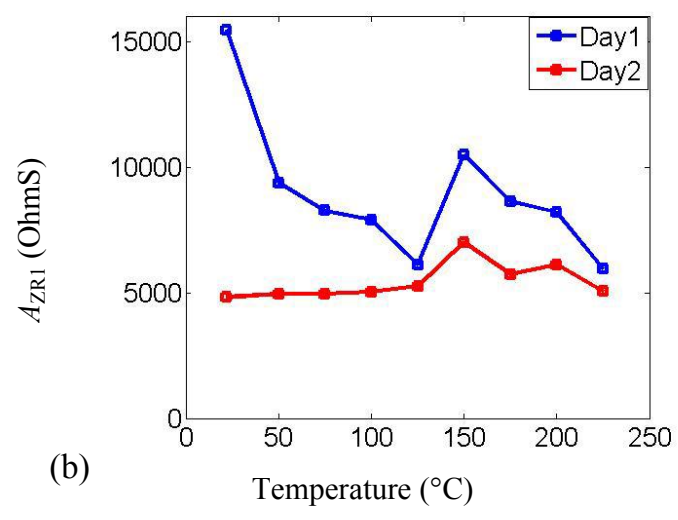

Figure 11.Change of amplitudes of the first resonance with temperature: (a) PWAS 1; (b) PWAS 2.

PWAS 1

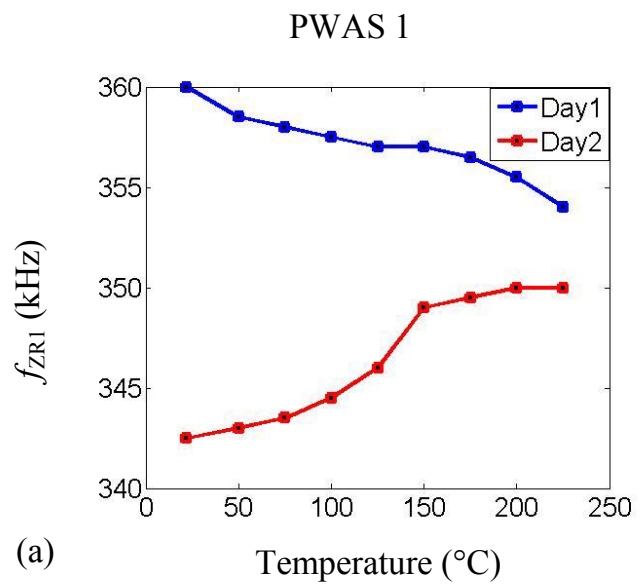

(a)

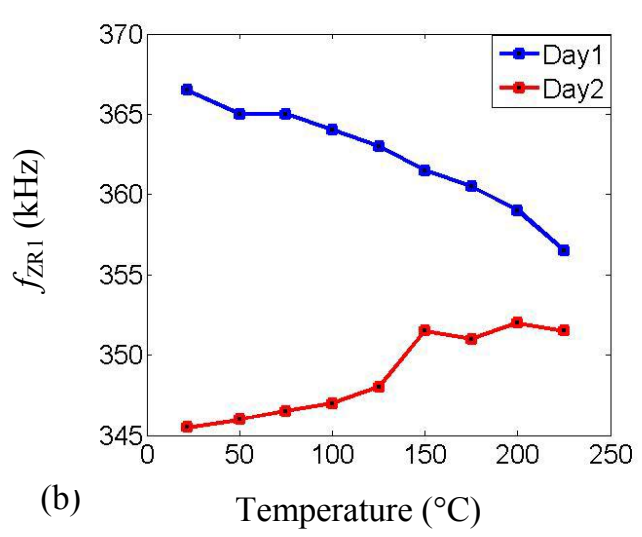

PWAS 2

Figure 12. Change of frequency of the first resonance with temperature: (a) PWAS 1; (b) PWAS 2

Table 1. Amplitude and frequency of first resonance at each temperature on Day 1

\begin{tabular}{|c|c|c|c|c|c|}
\hline & \multicolumn{2}{|l|}{ PWAS 1} & \multicolumn{2}{|l|}{ PWAS 2} \\
\hline & & $\mathrm{A}_{\mathrm{R} 1}(\mathrm{mS})$ & $\mathrm{f}_{\mathrm{R} 1}(\mathrm{kHz})$ & $\mathrm{A}_{\mathrm{R} 1}(\mathrm{mS})$ & $\mathrm{f}_{\mathrm{R} 1}(\mathrm{kHz})$ \\
\hline \multirow{9}{*}{ 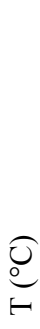 } & 22 & 11975 & 360.0 & 15492 & 366.5 \\
\hline & 50 & 8575 & 358.5 & 9376 & 365.0 \\
\hline & 75 & 7457 & 358.0 & 8278 & 365.0 \\
\hline & 100 & 7752 & 357.5 & 7922 & 364.0 \\
\hline & 125 & 7189 & 357.0 & 6118 & 363.0 \\
\hline & 150 & 8197 & 357.0 & 10504 & 361.5 \\
\hline & 175 & 7140 & 356.5 & 8653 & 360.5 \\
\hline & 200 & 6136 & 355.5 & 8197 & 359.0 \\
\hline & 225 & 5664 & 354.0 & 5965 & 365.5 \\
\hline
\end{tabular}


Table 2. Amplitude and frequency of first resonance with temperature (Day2)

\begin{tabular}{|c|c|c|c|c|c|}
\hline & \multicolumn{2}{|l|}{ PWAS 1} & \multicolumn{2}{|l|}{ PWAS 2} \\
\hline & & $\mathrm{A}_{\mathrm{R} 1}(\mathrm{mS})$ & $\mathrm{f}_{\mathrm{R} 1}(\mathrm{kHz})$ & $\mathrm{A}_{\mathrm{R} 1}(\mathrm{mS})$ & $\mathrm{f}_{\mathrm{R} 1}(\mathrm{kHz})$ \\
\hline \multirow{9}{*}{ 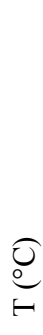 } & 22 & 4325 & 342.5 & 4821 & 345.5 \\
\hline & 50 & 4599 & 343.0 & 4957 & 346.0 \\
\hline & 75 & 4459 & 343.5 & 4971 & 346.5 \\
\hline & 100 & 4497 & 344.5 & 5042 & 347.0 \\
\hline & 125 & 4826 & 346.0 & 5271 & 348.0 \\
\hline & 150 & 6035 & 349.0 & 7026 & 351.5 \\
\hline & 175 & 5297 & 349.5 & 5746 & 351.0 \\
\hline & 200 & 5005 & 350.0 & 6126 & 352.0 \\
\hline & 225 & 3726 & 350.0 & 5067 & 351.5 \\
\hline
\end{tabular}

\section{SUMMARY AND CONCLUSIONS}

The development of active and passive nondestructive evaluation methods based on SHM provides an opportunity to progress the capability of monitoring DCSS and similar PVP systems. The integration of SHM with existent NDE tools in nuclear fields can increase the confidence of the safe operation and provide assurance of in-service reliability. Moreover, the development of SHM technologies can minimize human intervention, decrease the cost associated with NPP operation, and improve the reliability of essential systems by continuously assessing the structural integrity of nuclear related facilities. While SHM have been employed in different fields, its applicability for PVP systems will require further development and evaluation. This will required research to address some of the challenges that were discussed.

In this paper we have studied the free PWAS electromechanical impedance response to temperature elevation experimentally. Analytical electromechanical impendence mode is also provided in order to have a theoretical understanding of this PWAS parameter. In the experimental investigation, a 2-day temperature tests were conducted changing from room temperature to $225{ }^{\circ} \mathrm{C}$ with a step size of $25{ }^{\circ} \mathrm{C}$. Impendences in the range of $200 \mathrm{kHz}$ to $1 \mathrm{MHz}$ with a step size of $0.5 \mathrm{kHz}$ were measured at each temperature. The results led to the conclusion that the first two resonances within this frequency range shifted with the temperature change. The impendence change trends of the two PWAS being tested agreed with each other. Further analysis on the first resonance indicated that on the first day the amplitude gradually decreased; yet on the second day, the amplitudes increased before $150{ }^{\circ} \mathrm{C}$ and then decreased. The first resonance frequency also behaved differently for Day 1 and Day 2. On the first day the first resonance decreased monotonically and nearly linearly with the increase of the temperature while the frequencies increased on the second day. Further work is planned to study the different impedance behaviors between days with more PWAS and with extended duration.

\section{ACKNOWLEDGMENT}

The authors would like to acknowledge the financial support from Department of Energy award DE-NE0000726.

\section{REFERENCES}

[1] Giurgiutiu, Victor, Andrei Zagrai, and Jing Jing Bao. "Piezoelectric wafer embedded active sensors for aging aircraft structural health monitoring,"Structural Health Monitoring.Papers 1(1), 41-61 (2002).

[2] Ihn, Jeong-Beom, and Fu-Kuo Chang, "Pitch-catch active sensing methods in structural health monitoring for aircraft structures," Structural Health Monitoring.Papers 7(1), 5-19 (2008).

[3] Giurgiutiu, V; Gresil, M; Lin, B; Cuc, A; She Y and Roman.C, "Predictive Modeling of Piezoelectric Wafer Active Sensors Interaction with High-frequency Structural Waves and Vibration," Acta Mechanica. Papers 223, 1681-1691 (2012) 
[4] Alleyne, D.N. "The Nondestructive Testing of Plates Using Lamb Waves," Mechanical Engineering Department, Imperial College of London, London, (1991).

[5] Yu, L., Momeni, S., Godinez, V., Giurgiutiu, V., Ziehl, P., Yu, J., "Dual Mode Sensing with Low-Profile Piezoelectric Thin Wafer Sensors for Steel Bridge Crack Detection and Diagnosis", Advances in Civil Engineering. Papers 2012, article ID 402179(2012).

[6] Yu, Lingyu, and Victor Giurgiutiu, "In situ 2-D piezoelectric wafer active sensors arrays for guided wave damage detection," Ultrasonics. Papers 48(2), 117-134 (2008).

[7] Xu, B., Giurgiutiu, V., "Single mode tuning effects on Lamb wave time reversal with piezoelectric wafer active sensors for structural health monitoring," Journal of Nondestructive Evaluation.Papers 26(2-4), 123-134(2007).

[8] Finlayson, Richard D., et al, "Health monitoring of aerospace structures with acoustic emission and acoustoultrasonics," Insight-Wigston Then Northhampton. Papers 43(3), 155-158 (2001).

[9] Chaki, S., and G. Bourse., "Guided ultrasonic waves for non-destructive monitoring of the stress levels in prestressed steel strands," Ultrasonics. Papers 49(2), 162-171(2009).

[10] Gresil, Matthieu and et al, "Predictive modeling of electromechanical impedance spectroscopy for composite materials," Structural Health Monitoring. Papers 11(6), 671-683(2012).

[11] Lin, B., Giurgiutiu, V., Pollock, P., Xu, B., Doane, J., "Durability and Survivability of Piezoelectric Wafer Active Sensors on Metallic Structures," AIAA Journal, Papers 48(3), 635-643(2010A).

[12] Lin, B., Mendez-Torres, A. E., Gresil, M., Giurgiutiu, V., "Structural Health Monitoring with Piezoelectric Wafer Active Sensors Exposed to Irradiation Effects," ASME 2012 Pressure Vessels and Piping Division Conference. PVP 2012-78848(2012). 